\title{
Proximity effect of vanadium on spin-density-wave magnetism in $\mathrm{Cr}$ films
}

\author{
E. Kravtsov* \\ Institut für Experimentalphysik/Festkörperphysik, Ruhr-Universität Bochum, D-44780 Bochum, Germany \\ and Institute of Metal Physics, 620219 Ekaterinburg, Russia \\ A. Nefedov, F. Radu, A. Remhof, and H. Zabel \\ Institut für Experimentalphysik/Festkörperphysik, Ruhr-Universität Bochum, D-44780 Bochum, Germany \\ R. Brucas and B. Hjörvarsson \\ Department of Physics, Uppsala University, Box 530, S-75121 Uppsala, Sweden
}

\author{
A. Hoser \\ Institut für Kristallographie, RWTH-Aachen, D-52056 Aachen, Germany and Institut für Festkörperforschung, \\ Forschungszentrum Jülich, D-52425 Jülich, Germany \\ S. B. Wilkins \\ European Commission, JRC, Institute for Transuranium Elements, D-76125 Karlsruhe, Germany \\ and European Synchrotron Radiation Facility, F-38000 Grenoble, France
}

(Received 2 March 2004; published 30 August 2004)

\begin{abstract}
The spin-density-wave (SDW) state in thin chromium films is well known to be strongly affected by proximity effects from neighboring layers. To date the main attention has been given to effects arising from exchange interactions at interfaces. In the present work we report on combined neutron and synchrotron scattering studies of proximity effects in $\mathrm{Cr} / \mathrm{V}$ films where the boundary condition is due to the hybridization of $\mathrm{Cr}$ with paramagnetic $\mathrm{V}$ at the interface. We find that the $\mathrm{V} / \mathrm{Cr}$ interface has a strong and long-range effect on the polarization, period, and the Néel temperature of the SDW in rather thick Cr films. This unusually strong effect is unexpected and not predicted by theory.

DOI: 10.1103/PhysRevB.70.054425

PACS number(s): 75.50.Ee, 75.70.Ak, 75.30.Fv, 61.10.Nz
\end{abstract}

\section{INTRODUCTION}

The spin-density-wave (SDW) magnetism in $\mathrm{Cr}$ has attracted much attention since its first discovery in $1958 .{ }^{1,2}$ Its bulk behavior is well established and considerable progress has also been made towards understanding of the SDW magnetism in alloys with other transition metals (see reviews ${ }^{3-6}$ and references therein). During the last decade this field has regained much momentum because of a shift of the main focus towards thin $\mathrm{Cr}$ films and multilayers. The SDW behavior in these systems was found to display new features, differing drastically from the bulk ones due to dimensional and proximity effects.

Bulk $\mathrm{Cr}$ is an itinerant antiferromagnet displaying an incommensurate SDW (ISDW) below the Néel temperature $T_{N}=311 \mathrm{~K}$. The SDW consists of a sinusoidal modulation of the amplitude of the antiferromagnetically ordered magnetic moments. The SDW is a linear wave, propagating always along $\{100\}$ directions in the bcc Cr lattice, while the wave vector is incommensurate with the $\mathrm{Cr}$ lattice periodicity. At the spin-flip transition temperature $T_{S F}=123 \mathrm{~K}$, the SDW polarization changes from longitudinal (LSDW) $\left(T \leqslant T_{S F}\right.$, magnetic moments aligned parallel to the wave propagation direction) to transverse (TSDW) ( $T \geqslant T_{S F}$, magnetic moments aligned perpendicular to the wave propagation direction). Elastic strains and chemical impurities in Cr may cause the SDW to become commensurate (CSDW). In some cases the CSDW phase may be stable at temperatures much higher than $311 \mathrm{~K}^{3,4}$ Generally, in bulk $\mathrm{Cr}$ the SDW exists in a polydomain state below the Néel temperature, with magnetic domains populating all possible $\{100\}$ directions.

Investigations of the SDW in thin $\mathrm{Cr}$ films were initially motivated by the significant interest in exchange coupled $\mathrm{Fe} / \mathrm{Cr}$ multilayers displaying a giant magnetoresistance effect. $^{7}$ Today, an extensive literature has evolved, which traces different aspects of the $\mathrm{Cr}$ magnetism in $\mathrm{Fe} / \mathrm{Cr}$ systems. ${ }^{8-11}$ From these studies it was concluded that the formation of SDW magnetism in thin $\mathrm{Cr}$ films is governed by a strong exchange coupling acting at the $\mathrm{Cr} / \mathrm{Fe}$ interface on the one hand and by the interface structure/disorder on the other hand. ${ }^{12-14}$ Similar observations have also been made for the SDW behavior in other $\mathrm{Cr}$ /ferromagnet systems, such as $\mathrm{Cr} / \mathrm{Ni}$ and $\mathrm{Cr} / \mathrm{Co}^{15}$

Although a complete understanding of the SDW magnetism in $\mathrm{Cr} /$ ferromagnetic systems has not been reached yet, attention has, in part, shifted to layered heterostructures of $\mathrm{Cr}$ in contact with nonmagnetic metals. Among others, the SDW state in $\mathrm{Cr} / \mathrm{Ag},{ }^{16} \mathrm{Cr} / \mathrm{Cu},{ }^{15}$ and $\mathrm{Cr} / \mathrm{V}^{17,18}$ has been investigated recently. In particular, the $\mathrm{Cr} / \mathrm{V}$ system promises to provide new physics via the hybridization between very similar Fermi surfaces and the tunability of the Fermi surface with hydrogen uptake in the vanadium host lattice.

It is well known that the SDW behavior is connected with the peculiar features of the Cr Fermi surface providing nesting vectors between electron and hole sheets of similar shape. ${ }^{19}$ Doping with $\mathrm{V}$ decreases the electron concentration, 
and, hence, the magnetic moment of Cr. This is opposite to doping with $\mathrm{Mn}$ atoms, which enhances the magnetic moment and drives $\mathrm{Cr}$ towards CSDW order. ${ }^{20}$ Comprehensive investigations of bulk $\mathrm{CrV}$ alloys ${ }^{21-23}$ have revealed a linear decrease of the Néel temperature with the V concentration, followed by a corresponding decrease in the SDW period. The effect was found to be so strong that a concentration of only 4 at. \% V is enough to suppress totally the SDW state in $\mathrm{Cr}$. The decrease of the $\mathrm{Cr}$ magnetic moment in dilute $\mathrm{CrV}$ alloys has recently been confirmed by Mössbauer spectroscopy studies with ${ }^{119} \mathrm{Sn}$ probe atoms. ${ }^{24}$

From the above-mentioned facts it is reasonable to expect a strong suppression of the $\mathrm{Cr}$ magnetic moment and the SDW near the $\mathrm{Cr} / \mathrm{V}$ interface. Recent theoretical calculations of the $\mathrm{Cr} / \mathrm{V}$ interface magnetic structure performed by different groups ${ }^{25-29}$ confirm this notion. In addition, the calculations predict that the boundary $\mathrm{V}$ monolayer should gain an induced magnetic moment. Bihlmayer et al. found that a single $\mathrm{V}$ layer on $\mathrm{Cr}$ couples antiferromagnetically to $\mathrm{Cr}$ with an induced $\mathrm{V}$ moment of $2.1 \mu_{B}$. This rather large moment is due to surface states and would probably not develop in a thin film. Nevertheless, the induced moment in V is similar to the one found at the $\mathrm{Fe} / \mathrm{V}$ interface, where theoretical and experimental work established a V moment of about $1 \mu_{B}$, polarized opposite to the Fe moments. ${ }^{30-34}$ The interface effects discussed so far are expected to be of short range and should damp out quickly at a distance of several monolayers from the interface. In $\mathrm{Cr} / \mathrm{V}$ multilayers the damping effect should manifest itself by locating the node of the SDW in the vicinity of the $\mathrm{Cr} / \mathrm{V}$ interfaces. ${ }^{35}$ It should be noted, however, that all theoretical investigations assume that the interface is perfectly sharp and that the propagation of the SDW is in the direction normal to the interface. The propagation direction of the SDW is not a result of the various $a b$ initio calculations, but has been incorporated artificially.

Mibu et al. ${ }^{17,18}$ have recently provided experimental support for the outlined theoretical picture in a series of elegant experiments with Mössbauer spectroscopy by inserting the ${ }^{119} \mathrm{Sn}$ probe layers in $\mathrm{Cr} / \mathrm{V}$ multilayers at different distances from the interfaces. They have given direct experimental evidence for a reduction of the $\mathrm{Cr}$ magnetic moment near the interface region. $\mathrm{Cr}$ was found nonmagnetic at distances up to $20 \AA$ away from the interface, while at larger distances of about $40 \AA$ the $\mathrm{Cr}$ magnetic moment increases again and reaches values comparable to the bulk moment.

So far we have discussed the local and short range effects which occur close to the $\mathrm{Cr} / \mathrm{V}$ interface. Next we draw our attention to the more global properties. For instance, it would be highly interesting to investigate whether the $\mathrm{Cr} / \mathrm{V}$ proximity effect changes the Néel temperature and the SDW parameters, including period, polarization, and propagation direction. Some theoretical work points to these more global effects. According to Refs. 36 and 37, surface effects in isolated $\mathrm{Cr}$ films may extend up to several thousands of angstroms. In a similar fashion the $\mathrm{Cr} / \mathrm{V}$ interface may perturb the Cr SDW magnetism more extensively than assumed so far. This has to be verified by experiments.

In the present paper we address the above questions concerning the global perturbation of the Cr SDW magnetism by proximity effects at the $\mathrm{Cr} / \mathrm{V}$ interface. By proximity effects we understand any change of the SDW state induced by the interface of $\mathrm{Cr}$ with another material. We provide a systematic study of the SDW state in $2000 \AA$ thick $\mathrm{Cr}(001)$ films grown on a $14 \AA$ thick V(001) buffer layer. The Cr thickness is chosen to be large enough to ignore any local interface effects and to concentrate only on long-range effects arising from the $\mathrm{Cr} / \mathrm{V}$ interface. The amount of $\mathrm{V}$ atoms in the $\mathrm{V}$ layer is comparable with a $\mathrm{CrV}$ alloy of corresponding $\mathrm{V}$ concentration. From neutron and synchrotron scattering experiments we establish the magnetic phase diagram of the system and reveal the $\mathrm{Cr} / \mathrm{V}$ proximity effect on the SDW state in comparison to other $\mathrm{Cr}$ thin film systems, bulk $\mathrm{Cr}$, and $\mathrm{CrV}$ alloys.

\section{GROWTH AND SAMPLE CHARACTERIZATION}

The sample was grown with a UHV magnetron sputtering system on a $20 \times 20 \mathrm{~mm}^{2} \mathrm{MgO}(001)$ substrate. The deposition started with a V buffer layer of $14 \AA$ thickness and continued with a $2000 \AA$ thick Cr film. No protection layer was deposited, so a thin oxide layer is formed at the top of the Cr film. ${ }^{38}$ During the deposition the substrate temperature was kept at $200{ }^{\circ} \mathrm{C}$. After the preparation the sample was cut into several parts to be used in different experiments. In particular, we used a $15 \times 20 \mathrm{~mm}^{2}$ sample for neutron measurements and a $5 \times 5 \mathrm{~mm}^{2}$ sample for $\mathrm{x}$-ray experiments.

The structural information on the sample was obtained with X-ray diffraction studies done at the W1.1 and D3 beamlines of the HASYLAB, Germany. The measurements were performed at room temperature using a wavelength $\lambda$ $=1.5404 \AA$. The in- and out-of-plane lattice parameters of the $\mathrm{Cr}$ film as well as the epitaxial relationship between the film and $\mathrm{MgO}$ substrate were established precisely by analyzing the scattered intensity around the $\mathrm{Cr}(002)$ and (011) fundamental peaks. The epitaxial relation between $\mathrm{Cr}$ and $\mathrm{MgO}$ was confirmed to be $\mathrm{Cr}(001)[100] / / \mathrm{MgO}(001)[110]$ that is also valid for growth of $\mathrm{Cr}$ directly on the $\mathrm{MgO}(001)$ substrate without any buffer layer, ${ }^{39}$ which is equivalent with the well known $45^{\circ}$ epitaxial relation observed for $\mathrm{Fe}(001)$ on $\mathrm{MgO}(001)$. The $\mathrm{Cr}$ lattice was found to have a slight tetragonal distortion determined by the difference between the out-of-plane lattice parameter $a^{\perp}=2.8865 \pm 0.0005 \AA$ and the in-plane lattice parameter $a^{\|}=2.879 \pm 0.001 \AA$.

Next we discuss the structural correlation length and the film mosaicity. In Fig. 1(a) is shown the radial x-ray scan through the $\mathrm{Cr}(002)$ reflection. The solid line represents a fit to the data points by using a single Gaussian line shape. The full width at half maximum (FWHM) is estimated to be $\Delta(2 \theta)=0.044^{\circ}$. According to the Scherrer equation, ${ }^{40}$ this FWHM value yields an out-of-plane structural coherence length of $2000 \AA$, which corresponds exactly to the total $\mathrm{Cr}$ film thickness. In Fig. 1(b) is presented the transverse x-ray scan through the $\mathrm{Cr}(002)$ reflection. The solid line represents a fit to the data points by using a single Gaussian line shape. The FWHM is an estimate of the mosaic spread in the $\mathrm{Cr}$ film, which is about $0.10^{\circ}$. This is five times less than reported earlier for $\mathrm{Cr}$ growth on $\mathrm{MgO}(001)^{39}$ and about two times less than $\mathrm{Cr}$ growth on a $\mathrm{Nb}(001)$ buffer layers. ${ }^{15}$ Thus 

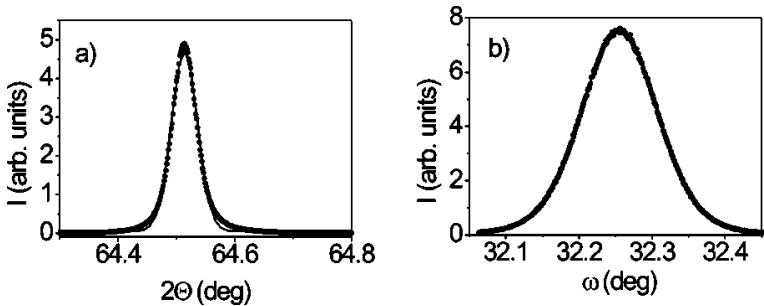

FIG. 1. (a) Radial x-ray scan through the $\mathrm{Cr}(002)$ reflection. The solid line is a fit to the data points with a single Gaussian line shape. The width of the peak depends on the Cr film thickness and reflects a $100 \%$ structural coherence of the film. (b) Transverse x-ray scan through the $\mathrm{Cr}(002)$ reflection. The solid line is a fit to the data points with a single Gaussian line shape with a FWHM of $0.10^{\circ}$

the initial growth of a thin $\mathrm{V}$ buffer layer turns out to improve the $\mathrm{Cr}$ intralayer structure significantly.

\section{SCATTERING EXPERIMENTS}

When dealing with $\mathrm{Cr}$ thin film systems, one should distinguish between in-plane and out-of-plane SDW propagation directions and polarizations that are nonequivalent due to the broken symmetry at the interfaces. The $\mathrm{Cr}$ magnetic structure can be explored by elastic neutron scattering providing complete information on the SDW state. The magnetic moment modulation produces corresponding satellite reflections around forbidden bcc Bragg reflections, which can be detected with neutron scattering.

The SDW is accompanied by a charge density wave (CDW) and a strain wave (SW), corresponding to periodic modulations of the charge density and the lattice spacing, respectively. The modulation period of the SW and CDW is half the period of the SDW. The SW can be investigated via $\mathrm{x}$-ray scattering, yielding information about the SDW period and propagation direction. ${ }^{41-43}$ Although SW investigations do not reveal the SDW polarization, they have the advantage of a higher reciprocal space resolution, which is important for a precise determination of the SDW period. The application of neutron and $\mathrm{x}$-ray scattering methods to the determination of the SDW parameters has been reviewed in some detail in a number of papers. We will not repeat this here and refer the interested reader to published papers and reviews for further information. ${ }^{3,13,15,44}$

\section{A. Synchrotron scattering measurements}

Synchrotron scattering experiments were performed at the ID20 magnetic scattering undulator beamline of the ESRF (Grenoble, France). ${ }^{45,46}$ The incident photon beam delivered by two phase undulators was $99.8 \%$ linearly $\sigma$-polarized in the sample plane. The primary slits in the optical system were set to utilize the first harmonic of the undulators. The incident beam energy was selected to be slightly below the $\mathrm{Cr}$ absorption edge of $5.989 \mathrm{keV}$ by using a $\mathrm{Si}(111)$ double crystal monochromator. The polarization of the scattered beam was analyzed by using a pyrolytic graphite $\mathrm{PG}(004)$ analyzer to select pure $\sigma \sigma$ scattered intensity, also providing a drastic reduction of the background signal. The measurements were taken at temperatures between 15 and $300 \mathrm{~K}$ by using a displex cryostat equipped with Be windows.

To determine the propagation direction of the SW and SDW we first performed two screening scans: one scan at the $\mathrm{Cr}(011)$ reflection in the $K$ direction to check possible inplane SW propagation, and one scan along the $L$ direction, crossing the $\mathrm{Cr}(002)$ reflection to search for out-of-plane SW. In Fig. 2 are shown the above scans measured at $15 \mathrm{~K}$ without polarization analysis. The fundamental (002) and (011) $\mathrm{Cr}$ peaks are removed from the figures for clarity as their intensity is many orders of magnitude higher than the intensity of the satellite reflections. In the panel on the right side
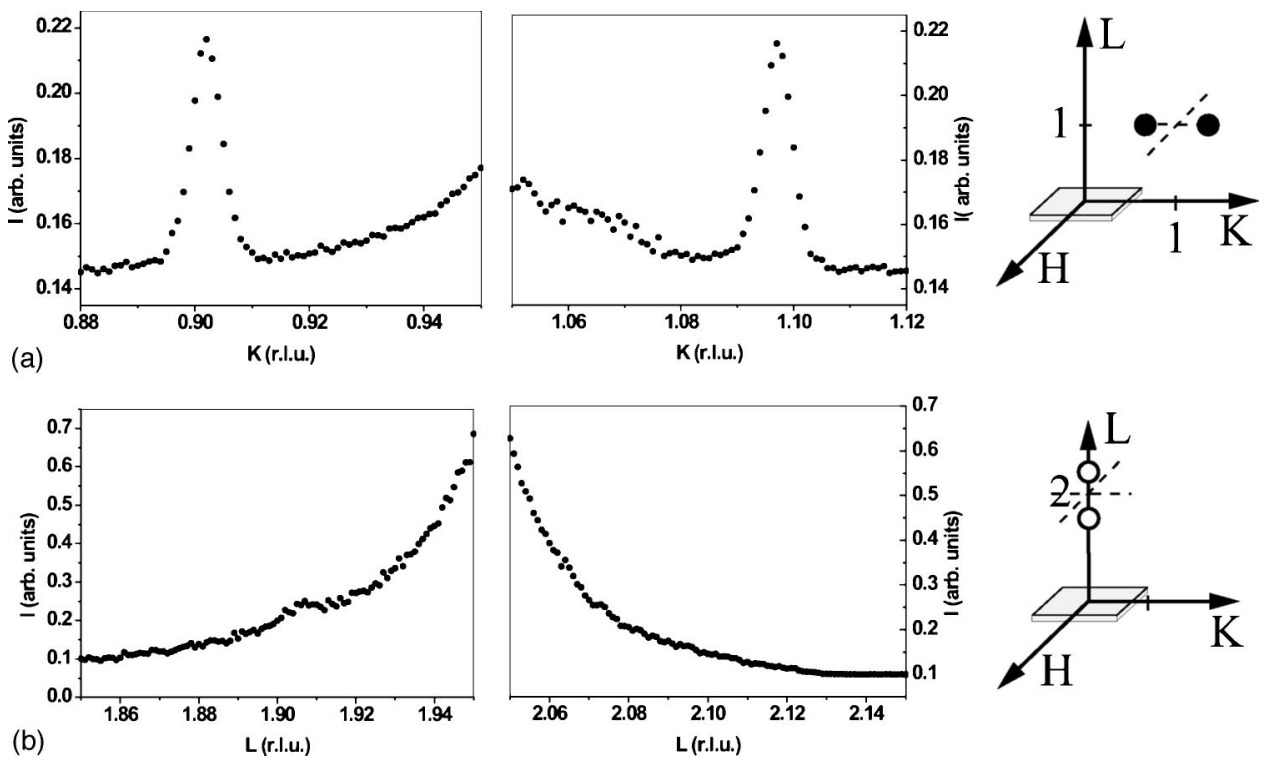

FIG. 2. Synchrotron radiation scans (a) along the $K$ direction around the $\operatorname{Cr}(011)$ peak and (b) along the $L$ direction around the $\mathrm{Cr}(002)$ reflection taken at $15 \mathrm{~K}$ without polarization analysis. The fundamental (002) and (011) Cr peaks are removed from the figures since their intensity is many orders of magnitude higher than the intensity of the satellite reflections. 

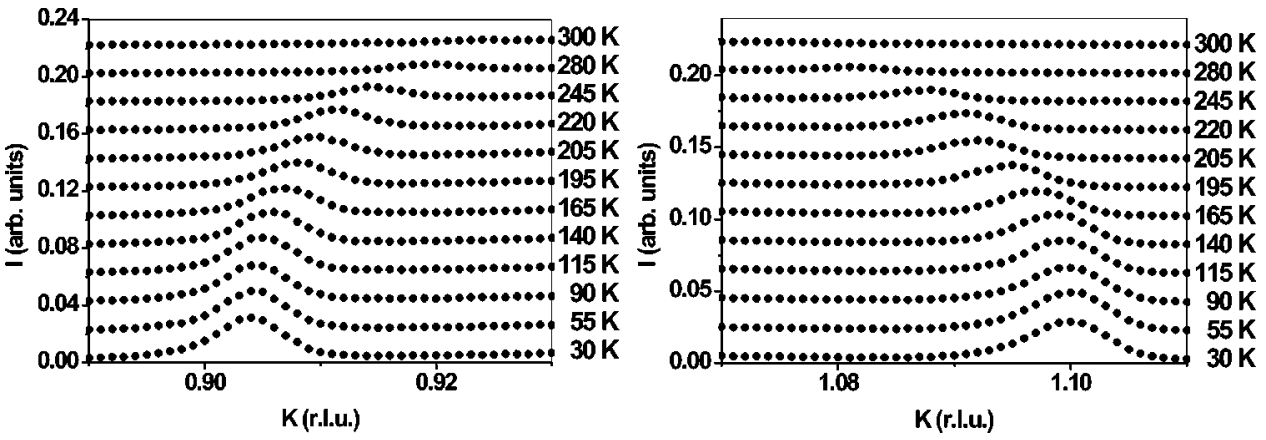

FIG. 3. Synchrotron measurement of the temperature dependence of the scattered intensity along the $K$ direction around the $\mathrm{Cr}(011)$ reflection taken with polarization analysis of the scattered beam. The fundamental $\mathrm{Cr}(011)$ peak is removed from the figure, the curves and the individual scans are shifted vertically by a constant factor for clarity.

the scan directions are indicated. The circles refer to satellite reflections allowed by the selection rules but not detected, whereas closed circles refer to allowed and detected satellites. From the recorded scans it is evident that in our case the strain wave propagates entirely in the film plane, whereas the out-of-plane wave is completely suppressed. In subsequent experiments we have analyzed in more detail the inplane SW component. In Fig. 3 we show the satellite's position and intensity measured for different temperatures in the range between 30 and $300 \mathrm{~K}$. As evident from the picture, the satellite's positions move smoothly towards the (011) peak as temperature increases, while the intensity decreases continuously with temperature. It is important to note that the FWHM of the satellite peaks, about 0.007 in r.l.u., is essentially independent of temperature over the entire temperature region investigated. At $300 \mathrm{~K}$ the satellite peak intensity has vanished, which can be taken as the Néel temperature of our system. The information on the SW behavior inferred from the synchrotron scattering data will be discussed further below together with the neutron scattering results.

\section{B. Neutron scattering measurements}

The neutron scattering experiments were performed on the triple-axis spectrometer UNIDAS installed at the FRJ-2 research reactor (Forschungszentrum Jülich, Germany). In the experiment we used the (002) reflection of a highly oriented pyrolytic graphite (PG) double crystal monochromator to select a neutron beam wavelength of $\lambda=2.351 \AA$. Another PG analyzer and a system of special slits and collimators were used to improve the instrumental resolution by reducing the neutron beam divergence and the background radiation. For these experiments the spectrometer was operated as a diffractometer by fixing the analyzer crystal to zero energy transfer. In this mode the background radiation is effectively filtered out. The contamination of the $\lambda / 2$ radiation was removed by placing a PG transmission filter in the incident neutron beam. The measurements were taken at temperatures between 30 and $300 \mathrm{~K}$ by using a displex cryostat with $\mathrm{Al}$ windows.

In Fig. 4 are presented neutron diffraction scans performed in the vicinity of the $\operatorname{Cr}(010)$ and $\operatorname{Cr}(001)$ positions in the reciprocal space. Above each set the scan directions are indicated. Open circles refer to satellite reflections allowed by the selection rules but not detected, whereas closed circles refer to allowed and detected reflections. The above neutron results are sufficient for a complete analysis of the SDW parameters including their temperature dependence. We note that the neutron scattering results are in complete agreement with the synchrotron scattering results. As seen in Fig. 4, the satellite reflections due to the incommensurate SDWs occur only in the $K$ direction in the vicinity of the (001) and (010) reflections. This implies that the SDW propagates in the film plane, as already conjectured from the $\mathrm{x}$-ray scans. The Néel temperature is estimated to be about $300 \mathrm{~K}$, which agrees with the synchrotron results. The temperature dependence of the SDW polarization is nearly identical to the one observed in bulk $\mathrm{Cr}^{47}$ At low temperatures we observe a longitudinal SDW. Above $100 \mathrm{~K}$ a spin-flip transition occurs to an in-plane transverse SDW. However, in contrast to the bulk behavior, the longitudinal and the transverse SDW are bidomain, and in both cases the $\mathrm{Cr}$ magnetic moments are aligned parallel to the film plane.

In addition to the satellite reflections from the ISDW phase, we also observe the (001) reflection corresponding to a CSDW. The commensurate phase coexists with the incommensurate one at all temperatures up to the Néel temperature. From Fig. 4 we notice that the intensity of the (001) reflection decreases smoothly with increasing temperature up to $300 \mathrm{~K}$, while the (010) peak intensity disappears above $150 \mathrm{~K}$. Thus the polarization of the CSDW is similar to the ISDW: the magnetic moments remain in the film plane for all temperatures. Below $100 \mathrm{~K}$ the magnetic moments are oriented parallel to the $H$ direction, and above $150 \mathrm{~K}$ parallel to the $K$ direction. The transition from one to the other polarization direction occurs continuously between 100 to $150 \mathrm{~K}$. The integrated intensity of the CSDW reflection is about $10 \%$ of that of the ICDW satellites. Both the CSDW and ISDW reflections have the same FWHM, which is directly connected with the in-plane SDW correlation length. The CSDW is usually due to strain effects. In the present system strain is provided by the pseudomorphic growth of the metal layers on the $\mathrm{MgO}$ substrate and by the mismatch of the respective thermal expansion coefficients.

In Fig. 5 is depicted the temperature phase diagram for the $\mathrm{V} / \mathrm{Cr}$ system. Both the ISDW and the CSDW propagate always in the film plane with the magnetic moments lying 

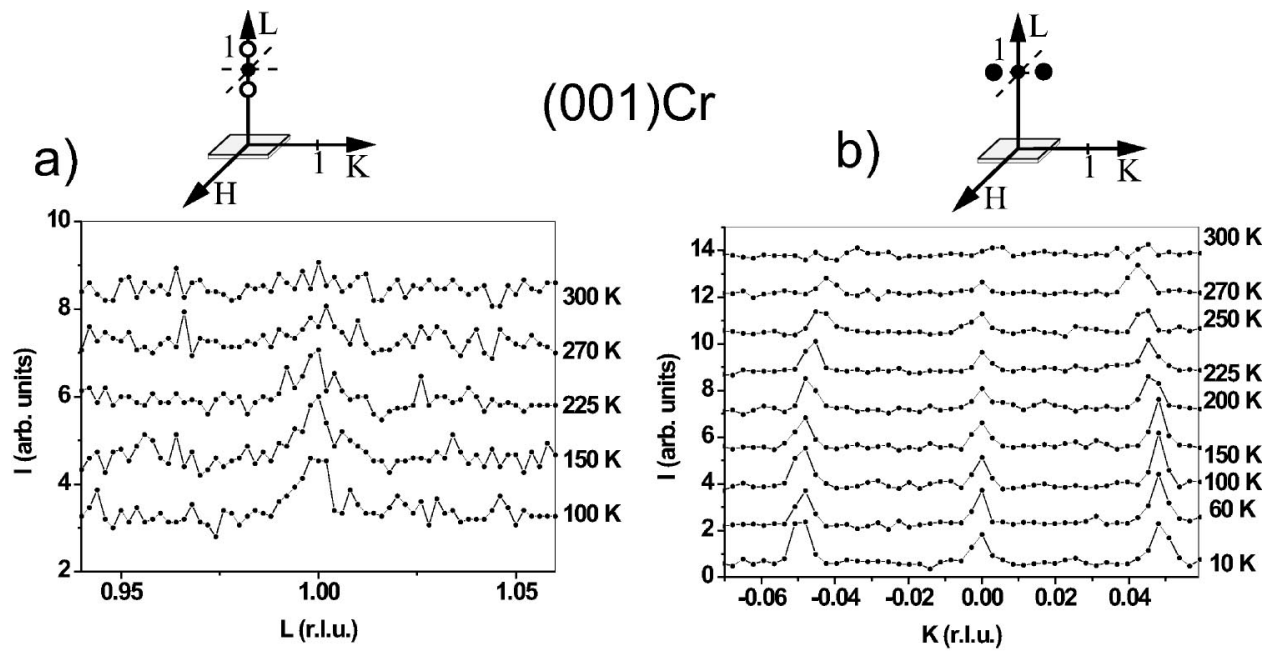

c)
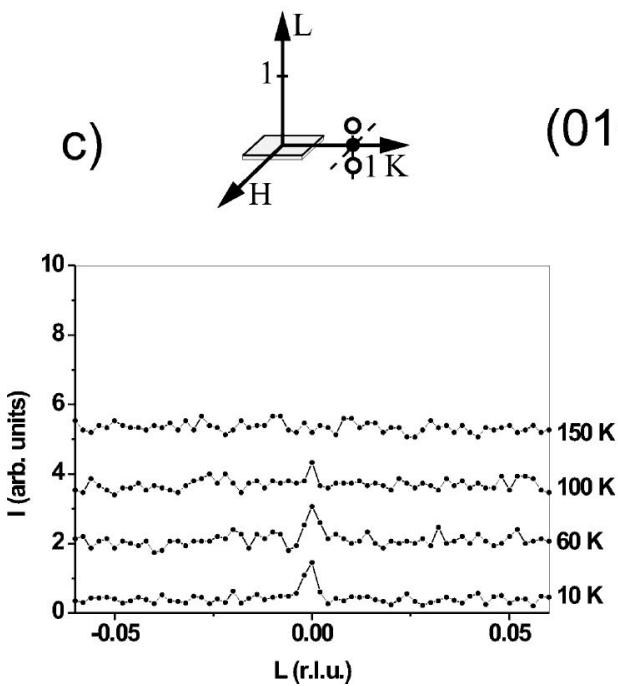

(010) $\mathrm{Cr}$

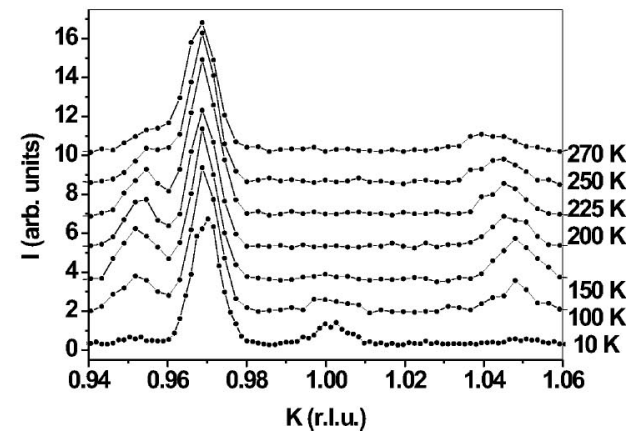

FIG. 4. Neutron scattering scans taken at the $\mathrm{Cr}(010)$ and the $\mathrm{Cr}(001)$ positions to explore the SDW polarization. The scans recorded at different temperatures are depicted with an offset in the vertical direction for clarity. The strong temperature-independent peak at $K$ $\sim 0.97$ r.l.u. in (d) is due to the $\mathrm{MgO}(022)$ reflection for the $\lambda / 2$ harmonics.

also in the film plane. The spin-flip transition for both phases starts at the same temperature of about $100 \mathrm{~K}$. While the spin-flip transition for the ISDW is more or less sharp, the spin reorientation transition for the CSDW is spread over a wide temperature interval of about $50 \mathrm{~K}$.

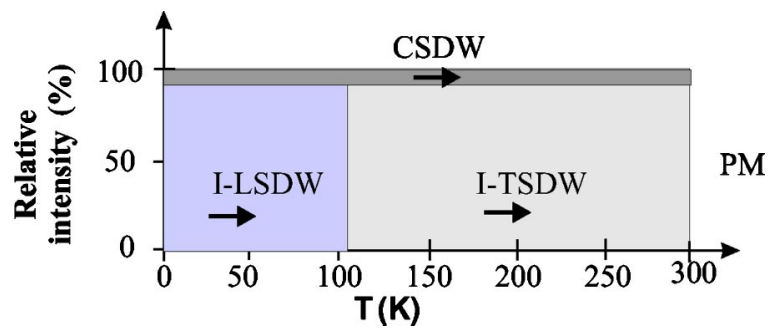

FIG. 5. Qualitative magnetic phase diagram for the spin density wave in a $2000 \AA$ thick $\mathrm{Cr}(001)$ film deposited on a $\mathrm{MgO}(001)$ substrate with a $14 \AA$ thick V buffer layer. The phase diagram is the result of combined synchrotron and neutron-scattering experiments, as described in the text.

\section{DISCUSSION}

The synchrotron and neutron scattering results described in the previous section allow us to draw a clear picture of the SDW behavior within the $\mathrm{Cr} / \mathrm{V}$ system. To work out the essential details arising from the $\mathrm{Cr} / \mathrm{V}$ proximity effect, we will compare the results obtained with those known from reference systems: bulk $\mathrm{Cr}$, dilute $\mathrm{CrV}$ alloy, and single $\mathrm{Cr}$ films on other paramagnetic or ionic substrates.

First we discuss possible effects of the vanadium buffer layer on the SDW polarization and propagation direction. It is generally recognized now that the SDW propagation direction in thin Cr films is governed by strain effects from substrate and buffer layers, whereas the SDW polarization is mainly due to proximity effects from neighboring layers. In case of a $4000 \AA$ thick $\mathrm{Cr}(001)$ film on a $\mathrm{Al}_{2} \mathrm{O}_{3}(1 \overline{102})$ substrate with a $\mathrm{Nb}(001)$ buffer layer a mixture of in-plane transverse and out-of-plane longitudinal ISDW has been observed below the Néel temperature, while for a $3000 \AA$ thick $\mathrm{Cr}$ film an out-of-plane LSDW is observed at low tempera- 
ture, which transforms to a out-of-plane TSDW at higher temperatures. ${ }^{48,49} \mathrm{In}$ all cases the $\mathrm{Cr}$ surface was uncapped and therefore was covered with a roughly $20 \AA$ thick $\mathrm{Cr}_{2} \mathrm{O}_{3}$ layer, which forms by natural oxidation. ${ }^{38}$ For a single $4500 \AA \mathrm{Cr}$ film grown on the $\mathrm{MgO}(001)$ substrate without any buffer layer, Kunnen et al. ${ }^{39}$ observed a mixture of CDSW and ISDW, which propagate in the film plane, while the spins are oriented in the direction normal to the film plane. Our neutron data for a $2000 \AA$ thick $\mathrm{Cr}$ film grown on $\mathrm{MgO}(001)$ substrate without buffer layer ${ }^{50}$ are in line with results of Kunnen et al. ${ }^{39}$ We found that the SDW propagates in the film plane, and that it has longitudinal polarization at temperatures below $100 \mathrm{~K}$ and transverse polarization with out-of-plane spins at temperatures from 100 to $270 \mathrm{~K}$. At higher temperatures solely a CSDW develops with in-plane propagation and out-of-plane spin orientation. In the present $\mathrm{Cr} / \mathrm{V}$ heterostructure the propagation direction agrees with that of the plain Cr film, but not the spin orientation. Therefore we conclude that the proximity effect at the $\mathrm{Cr} / \mathrm{V}$ interface is responsible for the spin reorientation from the out-ofplane to in-plane direction in the TSDW.

This unusually strong proximity effect is unexpected and it is difficult to provide here an adequate explanation. Based on present theoretical status of the field, we may speculate that the underlying mechanism is connected with the induced magnetic polarization at the $\mathrm{V} / \mathrm{Cr}$ interface. As mentioned in the Introduction, at $\mathrm{V} / \mathrm{Fe}$ and at $\mathrm{V} / \mathrm{Co}$ interfaces an induced $\mathrm{V}$ moment polarized antiparallel to the ferromagnetic moments has been established. ${ }^{31,32,51}$ It is possible that a similar polarization may also occur at the $\mathrm{V} / \mathrm{Cr}$ interface as it has been predicted theoretically. ${ }^{26} \mathrm{In}$ this case, the $\mathrm{V}$ magnetic moments are aligned parallel to the interface due to dipolar fields and are exchange coupled indirectly with the $\mathrm{Cr}$ magnetic moments across a nonmagnetic layer at the interface. ${ }^{17}$ This might explain the difference in spin orientation for $\mathrm{Cr}$ on plain $\mathrm{MgO}$ (out-of-plane) and for $\mathrm{Cr}$ on $\mathrm{MgO}$ with a thin $\mathrm{V}$ buffer layer (in-plane).

The actually observed SDW may not only depend on the electronic properties of the boundary layer, but also on the strain state and thickness of Cr. To the best of our knowledge, there is no theoretical prediction available as concerns the orientational effects caused by paramagnetic boundary layers. We note, however, that the effect of pinning the SDW nodes near the $\mathrm{Cr} / \mathrm{V}$ interfaces as predicted by $\mathrm{Hirai}^{35}$ does not apply in our case, since the SDW propagates in the layer plane.

Next we consider proximity effects of vanadium on other SDW parameters, in particular, on the SDW period. Since the synchrotron data on the SW are more precise than the neutron data, we use those to estimate the period of the SDW as a function of temperature. The data are plotted in Fig. 6 and compared to the SDW period in bulk Cr (Ref. 43) and in a $2000 \AA$ thick $\mathrm{Cr}$ film grown on a thick $\mathrm{Nb}$ buffer layer. ${ }^{48}$ Since no data are available for thin CrV films, we compare our results with a bulk $\mathrm{Cr}_{0.995} \mathrm{~V}_{0.005}$ alloy. ${ }^{52-54}$ This corresponds roughly to the same composition as $\operatorname{Cr}(2000 \AA) / \mathrm{V}(14 \AA)$ when mixed, which would result in a 0.7 at. \% alloy. As can be seen from the figure, the temperature dependence of the SW period in our $\mathrm{Cr} / \mathrm{V}$ film is similar to that in other $\mathrm{Cr}$ thin film systems and in bulk $\mathrm{Cr}$, but

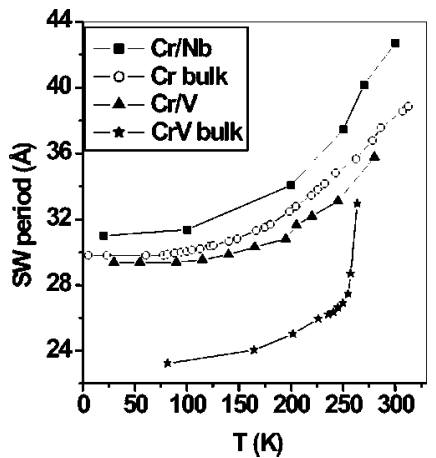

FIG. 6. Temperature dependence of the strain wave period in the Cr/V film and corresponding data for reference systems: $2000 \AA \mathrm{Cr}$ film on a thick $\mathrm{Nb}$ buffer layer (Ref. 48), bulk Cr (Ref. 43), and in bulk $\mathrm{Cr}_{0.995} \mathrm{~V}_{0.005}$ alloy (Ref. 52). The solid lines connecting the data points are guides to the eye.

completely different from that in the $\mathrm{Cr}_{0.995} \mathrm{~V}_{0.005}$ alloy. The value of the $\mathrm{SW}$ period in the $\mathrm{Cr} / \mathrm{V}$ film is smaller than in bulk $\mathrm{Cr}$ and in other thin $\mathrm{Cr}$ films, but much larger than in the corresponding bulk $\mathrm{CrV}$ alloy. To our knowledge, the SW period observed in our $\mathrm{Cr} / \mathrm{V}$ system is the smallest one observed so far in Cr-based thin films and multilayers.

In Fig. 7 we show the temperature dependence of the integrated intensity of the SW satellite peaks for the same systems. The integrated intensity $I(t)$ is proportional to the square of the order parameter which, in turn, is proportional to the $\mathrm{Cr}$ magnetic moment. Its temperature dependence is a characteristic of the phase transition from the antiferromagnetic to the paramagnetic state in $\mathrm{Cr}$. It is known that the temperature dependence $I(t)$ in bulk $\mathrm{Cr}$ can be described in analogy to the BCS theory of superconductivity. ${ }^{19}$

In the present $\mathrm{Cr} / \mathrm{V}$ system we find a quasilinear temperature dependence of $I(t)$ at low temperatures, which resembles the temperature dependence observed in $\mathrm{CrV}$ alloys,${ }^{52}$ but which is drastically different from the $I(t)$ dependence seen in bulk $\mathrm{Cr}$ and other thin films. Noakes et $a .^{52}$ speculated

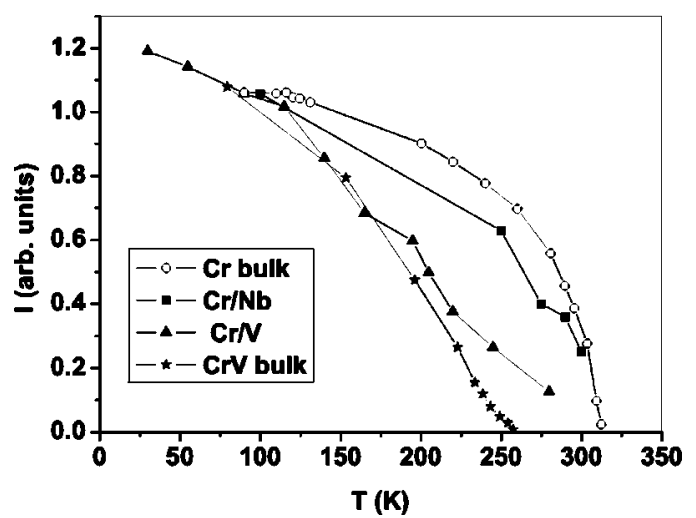

FIG. 7. Temperature dependence of the integrated intensity of the strain wave satellite reflections in the present $\mathrm{Cr} / \mathrm{V}$ film and corresponding data for reference systems: $2000 \AA \mathrm{Cr}$ film at thick $\mathrm{Nb}$ buffer (Ref. 48), bulk Cr (Ref. 43), and integrated intensity of the spin density wave satellite reflections measured with neutron scattering in bulk $\mathrm{CrV}$ alloy with a $\mathrm{V}$ concentration of 0.5 at. \% [from Noakes et al. (Ref. 52)]. 
that the unusual $I(t)$ behavior may not be an intrinsic feature of the $\mathrm{CrV}$ alloy, but due to strain effects. From our analysis it appears that the $I(t)$ dependence is an intrinsic property of the $\mathrm{CrV}$ systems. At higher temperatures the $I(t)$ for $\mathrm{Cr} / \mathrm{V}$ deviates from that of the $\mathrm{CrV}$ alloy and stretches towards the bulk Néel temperature. Another important difference is the temperature dependence for the FWHM of the SW satellite peaks in $\mathrm{Cr} / \mathrm{V}$ as compared to bulk $\mathrm{Cr}$. According to Hill et $a l .{ }^{43}$ in bulk $\mathrm{Cr}$ the FWHM of the SW satellite reflection increases with temperature, implying a reduction of the SDW correlation length with increasing temperature. In $\mathrm{Cr} / \mathrm{V}$ we find no change in the FWHM of the SW satellite peak over the entire temperature range and consequently no change in the correlation length.

\section{CONCLUSIONS}

We have studied the proximity effect of vanadium on the spin density wave magnetism in $\mathrm{Cr} / \mathrm{V}$ films. The sample was grown with a UHV magnetron sputtering system on $\mathrm{MgO}(001)$ substrate with a $14 \AA$ thick $\mathrm{V}(001)$ buffer layer. The $\mathrm{Cr}$ film exhibits a very high structural quality expressed by an out-of-plane coherence length corresponding to the film thickness and a very small mosaicity. The SDW properties were investigated by a combination of synchrotron scattering experiments to probe the strain waves and elastic neutron scattering to probe the spin density waves in the $\mathrm{Cr}$ film. It was shown that the $\mathrm{V}-\mathrm{Cr}$ hybridization at the interface causes a strong and long-range influence on the SDW behavior in the $\mathrm{Cr}$ film. First, we found that the $\mathrm{V}-\mathrm{Cr}$ interface hybridization changes the SDW polarization from out-ofplane to in-plane, i.e., the transverse incommensurate SDW propagates in the film plane and the magnetic moments are also in the plane. Second, for the SDW period and the order parameter we see a mixture of features which are typical for both bulk $\mathrm{CrV}$ alloys and simple $\mathrm{Cr}$ films. The temperature dependence of the SDW period is similar to that in other $\mathrm{Cr}$ films but different from the one in $\mathrm{CrV}$ alloys. On the other hand, the value of the SDW period is smaller than in bulk $\mathrm{Cr}$ and other $\mathrm{Cr}$ films, which is a characteristic feature of $\mathrm{CrV}$ alloys. The $\mathrm{Cr}$ magnetic moment in $\mathrm{Cr} / \mathrm{V}$ bilayers decreases quasilinearly at low temperatures, which is also observed in some $\mathrm{CrV}$ alloys but not in bulk $\mathrm{Cr}$ or other Cr-based thin film systems. The Néel temperature corresponds to the bulk value. Above the Néel temperature for the incommensurate phase we do not observe any commensurate SDW, which we ascribe to the high quality of the film. In any case, the commensurate SDW plays only a minor role in the present $\mathrm{Cr} / \mathrm{V}$ film system.

From the present experiments we conclude that the effect of a very thin V layer $(14 \AA)$ on a thick Cr film $(2000 \AA)$ is surprisingly large, changing drastically the global features of the SDW over the entire Cr film. This concerns the polarization of the SDW, the period, and the temperature dependence of the $\mathrm{Cr}$ magnetic moment at low temperatures. However, the Néel temperature is roughly the same as in the bulk.

\section{ACKNOWLEDGMENTS}

We would like to thank Dr. O. Seek and Dr. W. Morgenroth for help with the beamlines operation at the W1.1 and D3 instruments of the HASYLAB. This work has benefitted from collaborations within the Sonderforschungsbereich 491 "Magnetische Heteroschichten: Struktur und elektronischer Transport" funded by the Deutsche Forschungsgemeinschaft and from international collaborations supported by INTAS under Project No. 01-0386. E.K. acknowledges support from RFBR.
*Electronic address: evgeny.kravtsov@ruhr-uni-bochum.de

${ }^{1}$ L. Corliss, J.M. Hastings, and R.J. Weiss, Phys. Rev. Lett. 3, 211 (1959)

${ }^{2}$ V.N. Bykov, V.S. Golovkin, N.V. Agdeev, V.A. Ledvik, and S.I. Vinogradov, Dokl. Akad. Nauk SSSR 34, 1149 (1958).

${ }^{3}$ E. Fawcett, Rev. Mod. Phys. 60, 209 (1988).

${ }^{4}$ E. Fawcett, H.L. Alberts, V.Yu. Galkin, D.R. Noakes, and J.V. Yakhmi, Rev. Mod. Phys. 66, 25 (1994).

${ }^{5}$ J.J.M. Franse and R. Gersdorf, Landolt-Börnstein, New Series III/19A (Springer-Verlag, New York, 1986).

${ }^{6}$ H.P.J. Wijn, Landolt-Börnstein, New Series III/32A (SpringerVerlag, New York, 1997).

${ }^{7}$ M.N. Baibich, J.M. Broto, A. Fert, F. Nguyen Van Dau, F. Petroff, P. Etienne, B. Greuzet, A. Friederich, and J. Chazelas, Phys. Rev. Lett. 61, 2472 (1988)

${ }^{8}$ J. Unguris, R.J. Celotta, and D.T. Pierce, Phys. Rev. Lett. 69, 1125 (1992).

${ }^{9}$ E.E. Fullerton, K.T. Riggs, C.H. Sowers, S.D. Bader, and A. Berger, Phys. Rev. Lett. 75, 330 (1995).

${ }^{10}$ A. Schreyer, J.F. Ankner, Th. Zeidler, H. Zabel, M. Schäfer, J.A. Wolf, P. Grünberg, and C.F. Majkrzak, Phys. Rev. B 52, 16066 (1995); Europhys. Lett. 32, 595 (1995).
${ }^{11}$ P. Bödeker, A. Hucht, A. Schreyer, J. Borchers, F. Guthoff, and H. Zabel, Phys. Rev. Lett. 81, 914 (1998).

${ }^{12}$ D.T. Pierce, J. Unguris, R.J. Celotta, and M.D. Stiles, J. Magn. Magn. Mater. 200, 290 (1999).

${ }^{13}$ H. Zabel, J. Phys.: Condens. Matter 11, 9303 (1999).

${ }^{14}$ R.S. Fishman, J. Phys.: Condens. Matter 13, R235 (2001).

${ }^{15}$ P. Bödeker, A. Schreyer, and H. Zabel, Phys. Rev. B 59, 9408 (1999).

${ }^{16}$ S. Demuynck, J. Meersschaut, J. Dekoster, B. Swinnen, R. Moons, A. Vantomme, S. Cottenier, and M. Rots, Phys. Rev. Lett. 81, 2562 (1998).

${ }^{17}$ M. Almokhtar, K. Mibu, A. Nakanishi, T. Kobayashi, and T. Shinjo, J. Phys.: Condens. Matter 12, 9247 (2000).

${ }^{18}$ K. Mibu, M. Almokhtar, A. Nakanishi, T. Kobayashi, and T. Shinjo, J. Magn. Magn. Mater. 226-230, 1785 (2001).

${ }^{19}$ A.W. Overhauser, Phys. Rev. 128, 1437 (1962).

${ }^{20}$ E.E. Fullerton, J.L. Robertson, A.R.E. Prinsloo, H.L. Alberts, and S.D. Bader, Phys. Rev. Lett. 91, 237201 (2003).

${ }^{21}$ Y. Hamaguchi, E.O. Wollan, and W.C. Koehler, Phys. Rev. 138, A737 (1965).

${ }^{22}$ W.C. Koehler, R.M. Moon, A.L. Trego, and A.R. Mackintosh, Phys. Rev. 151, 405 (1966). 
${ }^{23}$ A.L. Trego and A.R. Mackintosh, Phys. Rev. 166, 495 (1968).

${ }^{24}$ S.M. Dubiel, J. Cieslak and F.E. Wagner, Phys. Rev. B 53, 268 (1996).

${ }^{25}$ A. Boussendel and A. Haroun, Thin Solid Films 325, 201 (1998).

${ }^{26}$ G. Bihlmayer, T. Asada, and S. Blügel, Phys. Rev. B 62, R11 937 (2000).

${ }^{27}$ B.A. Hamad and J.M. Khalifeh, Surf. Sci. 492, 161 (2001).

${ }^{28}$ A. Kellou, N.E. Fenineche, A. Tadjer, and H. Aourag, Mater. Chem. Phys. 80, 215 (2003).

${ }^{29}$ H.C. Herper, L. Szunyogh, P. Entel, and P. Weinberger, Phys. Rev. B 68, 134421 (2003).

${ }^{30}$ M.M. Schwickert, R. Coehoorn, M.A. Tomaz, E. Mayo, D. Lederman, W.L. O'Brien, Tao Lin, and G.R. Harp, Phys. Rev. B 57, 13681 (1998).

${ }^{31}$ A. Scherz, H. Wende, P. Poulopoulos, J. Lindner, K. Baberschke, P. Blomquist, R. Wappling, F. Wilhelm, and N.B. Brookes, Phys. Rev. B 64, 180407 (2001).

${ }^{32}$ A. Scherz, P. Poulopoulos, R. Nünthel, J. Lindner, H. Wende, F. Wilhelm, and K. Baberschke, Phys. Rev. B 68, 140401 (2003).

${ }^{33}$ D. Labergerie, K. Westerholt, H. Zabel, and B. Hjörvarsson, J. Magn. Magn. Mater. 225, 373 (2001).

${ }^{34}$ V. Uzdin, K. Westerholt, H. Zabel, and B. Hjörvarsson, Phys. Rev. B 68, 214407 (2003).

${ }^{35}$ K. Hirai, Phys. Rev. B 66, 132406 (2002).

${ }^{36}$ S.M. Dubiel and J. Cieslak, Phys. Status Solidi A 191, 577 (2002).

${ }^{37}$ J. Cieslak and S.M. Dubiel, Phys. Status Solidi A 196, 181 (2003).

${ }^{38}$ A. Stierle and H. Zabel, Europhys. Lett. 32, 365 (1997).

${ }^{39}$ E. Kunnen, S. Mangin, V.V. Moshchalkov, Y. Bruynseraede, A. Vantomme, A. Hoser, and K. Temst, Thin Solid Films 414, 262 (2002).

${ }^{40}$ B.E. Warren, X-ray Diffraction (Dover, New York, 1990).
${ }^{41}$ Y. Tsunoda, M. Mori, N. Kunotomi, Y. Teraoka, and J. Kanamori, Solid State Commun. 14, 287 (1974).

${ }^{42}$ D. Gibbs, K.M. Mohanty, and J. Bohr, Phys. Rev. B 37, 562 (1988).

${ }^{43}$ J.P. Hill, G. Helgesen, and D. Gibbs, Phys. Rev. B 51, 10336 (1995).

${ }^{44}$ H. Zabel, A. Schreyer, P. Bödeker, and P. Sonntag, in Dynamical Properties of Unconventional Magnetic Systems, Vol. 349 of NATO ASI Series E: Applied Sciences, edited by A.T. Skjeltorp and D. Sherrington (Kluwer, Dordrecht, 1998).

${ }^{45}$ A. Stunault, C. Vettier, F. de Bergevin, N. Bernhoeft, V. Fernandez, S. Langridge, E. Listrom, J.E. Lorenzo-Diaz, D. Wermeille, L. Chabert, and R. Chagnon, Synchrotron Radiat. 5, 1010 (1998).

${ }^{46}$ D. Mannix, P.C. de Camargo, C. Giles, A.J.A. de Oliveira, F. Yokaichiya, C. Vettier, Eur. Phys. J. B 20, 19 (2001).

${ }^{47}$ S.A. Werner, A. Arrott, and H. Kendrick, Phys. Rev. 155, 528 (1967).

${ }^{48}$ P. Sonntag, P. Bödeker, A. Schreyer, H. Zabel, K. Hamacher, and H. Kaiser, J. Magn. Magn. Mater. 183, 5 (1998).

${ }^{49}$ P. Sonntag, P. Bödeker, T. Thurston, and H. Zabel, Phys. Rev. B 52, 7363 (1995).

${ }^{50}$ E. Kravtsov, R. Brucas, B. Hjörvarsson, G. McIntyre, A. Nefedov, F. Radu, A. Remhof, and H. Zabel (unpublished).

${ }^{51}$ Y. Huttel, G. van der Laan, T.K. Johal, N.D. Telling, and P. Bencok, Phys. Rev. B 68, 174405 (2003).

${ }^{52}$ D.R. Noakes, T.M. Holden, P.C. de Camargo, E. Fawcett, and P. de V. DuPlessis, J. Appl. Phys. 64, 5883 (1988).

${ }^{53}$ P.C. de Camargo, A.J.A. de Oliveira, C. Giles, F. Yokaichiya, and C. Vettier, Mater. Sci. Forum 302-303, 33 (1999).

${ }^{54}$ A.J.A. de Oliveira, O.F. de Lima, W.A. Ortiz, and P.C. de Camargo, Solid State Commun. 96, 383 (1995). 